\title{
PERIODICAL ERRORS OF HIPPARCOS STAR ABSCISSAE
}

\author{
V. V. MAKAROV \\ Pulkovo Observatory \\ 196140 Saint-Petersburg \\ Russia
}

\begin{abstract}
An origin of both accidental and systematic errors of Hipparcos star abscissae is studied in the frames of the great circle reduction algorithm, adopted in the Hipparcos mission. Systematic variations of the basic angle within one turn of the Hipparcos satellite may be caused by periodical irregularities of the thermal flow from the sun radiation inside the satellite optical unit, enclosed in a hexagonal envelope. This would lead to periodical errors in the star abscissae at certain periods. It is shown, that an oscillation of the basic angle with a period of 60 degrees is the most dangerous one, as it is amplifyed 4.5 times. Lindegren et al. (1992) found, that the sixth harmonic in the star abscissae residuals clearly dominated with an amplitude of 0.96 milli-arcsec. If this is caused by basic angle disturbances, it follows that the amplitude of such variation may be up to 0.21 milli-arcsec. It is pointed out, that the thermal basic angle disturbances are not the only reason to expect periodical errors in star abscissae. Random abscissa errors too are inevitably of strong periodical sort, the periods of the largest harmonics being $60,30,18.9(2 \pi / 19), 14.4(2 \pi / 25)$ and $11.6(2 \pi / 31)$ degrees for a basic angle of 58 degrees. They arise from the spatial spectrum transformation of the white noise in the primary observational data due to so-called 'non-rigidity' of star abscissae. The non-rigidity term acts as a factor to the photon noise errors of observations for stars of certain magnitude. A radical way to diminish both accidental and systematic periodical errors in a future Hipparcos-like astrometric satellite would be to use two different basic angles (Makarov, 1992). The basic angles of 58 and 74 degrees were considered, as they were found to be an optimal combination. In that case an improvement of precision by a factor of 2.8 can be achieved with the same Hipparcos parameters, some part of which should be explained by doubling number of observations, that is $\sqrt{2}$. It may be concluded, that using two telescopes with different basic angles for space astrometry allows to increase significantly precision for a great deal of stars under the same conditions.
\end{abstract}

Acknowledgements: It is a pleasure to thank E. Høg and L. Lindegren for useful discussions of the problem. This work was supported by the Copenhagen University Observatory and the Danish Space Board.

\section{References}

Lindegren, L., van Leeuwen, F., Petersen, C., Perryman, M. A. C., Soderhjelm, S. (1992) 'Positions and parallaxes from The Hipparcos satellite. A first attempt at a global astrometric solution', Astron. and Astroph., 258, 134.

Makarov, V. V. (1992) 'Accidental periodical errors of the Hipparcos star abscissae', Pis'ma Astron. Zh. (Sov. Astron. Lett., in Russian), in press. 